\title{
Der Gehalt des Lichts an Ultraviolett. II.
}

(Zugleich Antwort auf die Entgegnung von Prof. Dorno in Bd. 103 dieses Archivs.)

Von

\section{Dr. Fritz Schanx,} presden.

Mit 2 Textabbildungen.

Meine lichtbiologischen Arbeiten haben von zwei Seiten Bestätigung erfahren. H. Pfeiffer und G. Beyer haben in ihrer Arbeit: Zur Kenntnis lichtkatalytischer Wirkungen ${ }^{1}$ ) sich eingehend mit der optischen Sensibilisation beschäftigt und dabei meine darauf bezüglichen Arbeiten weitgehend bestätigt und ergänzt. Eine weitere Bestätigung finden sie in den Arbeiten von $\mathrm{Burge}^{2}$ ), der während des Krieges in Amerika die Wirkungen des Lichtes auf die Augenlinse studierte. In Bd. 103 dieses Archivs hat meinen Untersuchungen über den Gehalt des Lichtes an Ultraviolett Prof. Dorno erneut widersprochen. In der gemeinsamen Sitzung der Berliner Ophthalmologischen Gesellschaft und der Deutschen Beleuchtungstechnischen Gesellschaft ist mir bei der Diskussion: Auge und Belichtung ${ }^{2}$ ) ein neuer Gegner erwachsen aus dem Kreise der Beleuchtungstechniker. Dr. Lux glaubte auch an der Hand physikalischer Energiemessungen meine Anschauung widerlegen zu können. Diese Widersprüche möchte ich aufklären.

Die Arbeit von Burge beschaftigt sich auch mit der Frage, welche Lichtstrahlen wirken auf die Augenlinse, und bestätigt meine Ansicht, daB die ultravioletten Strahlen imstande sind, das Linseneiweiß zu verändern. Die Arbeiten sind bei uns im Original schwer zugängig, und darum halte ich mich hier möglichst an den Bericht, den $\mathrm{Lux}$, mit dessen Untersuchungen ich mich später noch zu beschäftigen haben werde, von diesen Arbeiten gibt ${ }^{3}$ ).

Nach diesem Bericht fand Burge, ,daß es wesentlich die sehr kurzwelligen Strahlen zwischen 0,249 und $0,302 \mu$ mit einem Maximum bei $0,254 \mu$ sind, die a die Proteine koagulierend wirken.

$\left.{ }^{1}\right)$ Zeitschr. f. g. ges. exper. Med. 14, Hft. $3 / 4$.

2) Trans. III. Eng. Soe. (US.) 10 S. 932. 1915. Am. Journ. Physiolog. 36, 335. 1916; Electr. World 65, 912. 1915.

a) Dtsch. opt. Wochenschr. 1921, Nr, 34. 
Die Versuche von Burge erstreckten sich zunächst auf dünne Schichten von eingetrocknetem HühnereiweiB und zerquetschten Linsen von Froschaugen. Die Schichten blieben vollständig durchsichtig, auch wenn sie 100 Stunden hindurch von einer Quecksilberquarzlampe bestrahlt wurden; sie koagulierten an den von den Strahlen getroffenen Stellen aber sofort, wenn sie nach der Bestrahlung oder auch von vornherein mit gewissen Salzlösungen (vor allem Calciumund Magnesiumsalzen, dann aber auch Natrium- und Kaliumsilicaten) behandelt wurden. Sehr interessant und wichtig sind hierbei die spektrographischen Feststellungen Burges. Wurde das mit dem Quarzspektrographen zerlegte Licht der Quarzlampe auf die Proteinschicht geworfen, so bildeten sich die Spektrallinien als Streifen koagulierten Eiweißes ab, und zwar zwischen 0,249 bis $0,302 \mu$ mit einem ausgesprochenen Maximum bei $0,254 \mu$. Hierbei war es gleichgültig, ob die Proteinschicht mit den genannten Salzlösungen vorbehandelt oder in diesen Lösungen nachträglich analog einer photographischen Platte entwickelt wurde.

Die Versuche wurden dann auf lebende Organismen ausgedehnt, und zwar auf Fisch-und Froschaugen. Die Linsen dieser blieben selbst nach mehrmaliger, je sechsstündiger Bestrahlung durch die Quarzlampe aus unmittelbarer Nähe völlig klar, wenn die Individuen in reinem, möglichst salzfreicm Wasser lebten. Dagegen traten Trübungen und Kataraktbildung ein, wenn dem Aufenthaltwasser der Prüfobjekte nur 0,1\% Magnesium- oder Calciumsalz bzw. die gleiche Menge Natriumsilicat zugefügt würde.

Die Ansicht Burges, daß die Kataraktbildung bzw. die Koagulierung der Proteine immer nur dann eintrete, wenn in dem geschädigten Auge größere Mengen der genannten Salze vorhanden sind, wird durch seine Untersuchung an Tausenden von Linsenstar befallener Indier und Nordamerikaner gestützt. In dem Aschenrückstand dieser Linsen fanden sich immer nennenswerte Mengen der genannten Salze, bei den Indiern vor allem Silicate. Burge nimmt daher an, daß die schädigende Wirkung der kurzwelligen Strahlen immer eine Stoffwechselstörung zur Voraussetzung haben müsse, bei der ein Überreichtum der genannten Salze durch die Lymphbahnen nach der Linse transportiert wird. Bei den Indern erklärte er das Vorhandensein von Silicaten in der Linse durch das gewohnheitsmäßig geübte Tonessen. Es wäre von Wichtigkeit, nach dieser Richtung auch die Linsen von staroperierten Glasbläsern zu untersuchen, bei deren enormem Wasserkonsum infolge starken Schwitzens beträchtliche Kalkaufnahme stattfindet.

Diese Untersuchungen zeigen deutlich, daB es die ultravioletten Strahlen sind, die die Linse schädigen. Daß diese an sich schon das Linseneiweiß schädigen, ist eingangs erwähnt, die Anwesenheit von 
gewissen Salzen steigert deren Wirkung. Ob diese Salze in gleicher Weise wirken wie die Sensibilisatoren, mit denen ich gearbeitet, kann bei diesen Ausführungen unberücksichtigt bleiben. Die Feststellung Burges, daß es die ultravioletten, nicht die sichtbaren und ultraroten Strahlen sind, die das Linseneiweiß schädigen, stützt meine Ansicht und zeigt wieder, daß wir den Gehalt des Ultraviolett im Tageslicht. studieren müssen.

Die Physik der Sonnenstrahlung ist schon seit längerer Zeit der Gegenstand eingehender Studien. Wie weit ist der Gehalt des Sonnenlichtes an Strahlen erforscht, die direkt auf die Linse und auf die lebende Substanz einzuwirken vermögen? Die ausfübrlichste systematische Erforschung der Sonnenstrahlung wird ausgeführt nach der Methode, die Langley erdacht, und die seine Schüler und Nachfolger, Abbot, Fowle und Aldrich mit großer Experimentierkunst und technischer Geschicklichkeit, fußend auf Millionenstiftungen amerikanischer Mäzene, ausgebaut, nunmehr schon über $11_{2}$ Jahrzehnt an verschiedenen Orten der Erde und in verschiedenen Höhenlagen angewandt. Die Hauptbeobachtungen sind auf dem $1730 \mathrm{~m}$ hohen Mount Wilson in Kalifornien angestellt, viel ist auch in Washington fast in Neereshöhe gemessen, andererseits sind $4410 \mathrm{~m}$ Meereshöhe auf dem Mount Whitney in Kalifornien erklommen und zu Kontrollzwecken sind zwei Expeditionen ausgeführt worden nach Algier (Bassour, $1160 \mathrm{~m}$ Meereshöhe) in eine Entfernung von einem Drittel des Erdumfangs vom gleichzeitigen Beobachtungsort, dem Mount Wilson.

Diesen Untersuchungen liegt als Einheitsmaß für die Sonnenstrahlung das der Wärme zugrunde, in der Annahme, da $B$ alle Strahlen, die kurzwelligen wie die langwelligen, demjenigen Körper, der sie restlos absorbiert, eine ihrer Energie entsprechende Wärmemenge zuführen. $\mathrm{Zu}$ diesen Messungen konstruierte Langley sein Bolometer, welches von seinem Nachfolger $A b b o t$ weiter ausgebaut wurde. Eine genauere Beschreibung findet sich in Dorno, Physik der Sonnen- und Himmelsstrahlung. Mit diesem Instrument mißt man spektral die Energie der Sonnenstrahlen und rühmt sich, bis zur 3. Dezimale genau den Energiewert der Sonne in Wärmeeinheiten, die sog. Solarkonstante, berechnen zu können. Durch derartige Messungen erhält man als das normale Sonnenspektrum die Kurve, wie sie in Abb. 1 wiedergegeben ist. Darnach hat im terrestrischen Sonnenspektrum bei mittlerer Sonnenhöhe das Ultrarot etwa $60 \%$, das sichtbare Spelstrum etwa $40 \%$ Anteil an der Gesamtenergie, und das Ultraviolett nur noch kaum 1\%. Bei niedrig sinkender Sonne schwindet der letztere Teil immer mehr bei gleichzeitigẹ sehnellerer Zunahme des ultraroten Teils.

In der Physik mißt man auch auf dieselbe Weise die spektrale Energieverteilung in der Strahlung des absolut schwarzen Körpers bei 
verschiedenen Temperaturen. Die Abb. 2 zeigt solche Kurven, sie ist entlehnt den Ausführungen, die Lux in der gemeinsamen Sitzung der Berliner Ophthalmologischen Gesellschaft und der Deutschen Be-

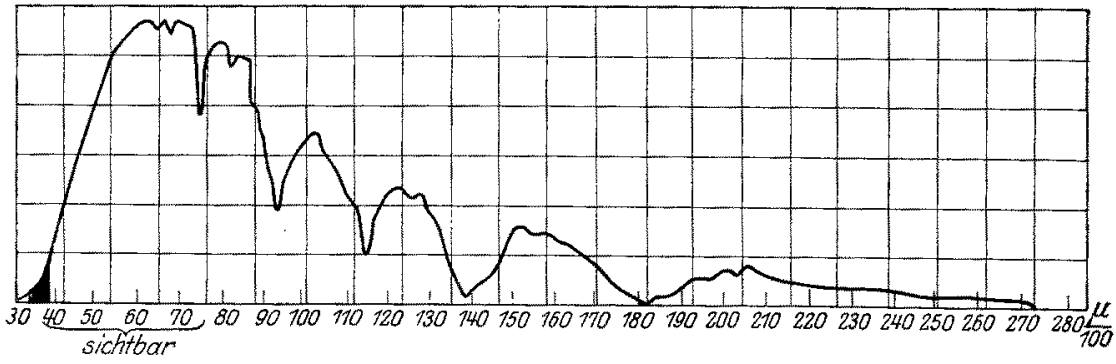

Abb. 1. Normales Sonnenspektrum. (Nach $A$ b b o t.)

leuchtungstechnischen Gesellschaft gemacht hat ${ }^{1}$ ). An der Kurve, welche der Temperatur des schwarzen Körpers von $4200^{\circ}$ entspricht, sind die Verhältnisse be. sonders deutlich zu erkennen. Die von der Kurve und der Abszissenachse eingeschlossene Fläche ist ein direktes Maß für die gesamte gestrahlte Energiemenge. Das in der linken Ecke schwarz gezeichnete kleine Flächenstückehen entspricht der im ultravioletten Gebiet gestrahlten Energiemenge, sie ist nur ein versch windender Bruchteil, etwa $2,4 \%$ der Gesamtenergie. Bei niedriger Temperatur ändert sich das Verhältnis noch weiter zuungunsten des ultravioletten Strahlungsteiles. Bei einer Temperatur von $2000^{\circ}$, die noch erheblich über der in unseren Hoch-

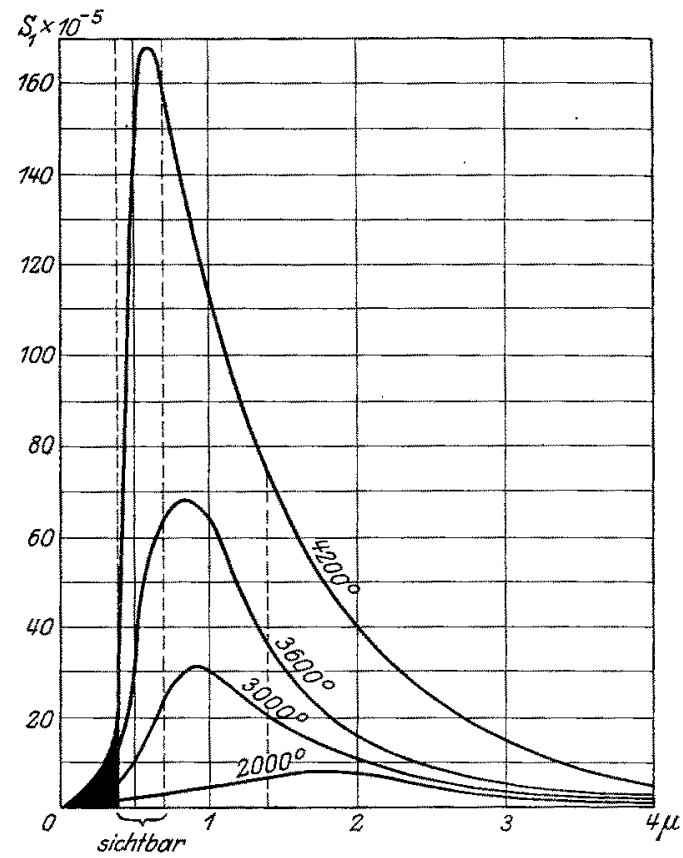

Abb. 2. Spektrale Energieverteilung des sehwarzen Korpers bei verschiedenen Temperaturen. (Nach L in x.) öfen, Flammenöfen, Glaswannenöfen usw. liegt, ist der ultraviolette Anteil der Strahlung kaum mehr zu messen. Auf der Zeichnung fällt dieser Anteil schon fast mit 1) Dtsch. opt. Wochenschr. 1921. 
der Abszissenachse zusammen. Er beträgt höchstens 0,05\%. Da die Temperatur unserer gebräuchlichen Lichtquellen zwischen 1700 und $2745^{\circ}$ liegt, wenn wir einerseits die Stearin- oder Paraffinkerze, andererseits die Gasfüllungslampe ins Auge fassen, so kann man nach der Ansicht von Lux wohl kaum behaupten, daß die ultraviolette Strahlung bei unseren künstlichen Lichtquellen dem Quantum nach irgendeine belangreiche Rolle spielt. Seiner Ansicht nach sind in den Strahlen einer Kohlenfadenlampe, dem Energiequantum nach, kaum mehr ultraviolette Strahlen vorhanden als in dem reflektierten. Licht von einem Kornblumenstrau $ß$ an einem leuchtenden Sommersonnentag.

Das sind Feststellungen von Physikern über den ultravioletten Anteil an der Strahlung des Lichtes. Allen lichtbiologischen Vorgängen liegen bekanntlich chemische Veränderungen zugrunde. Nur die kurzwelligen Strahlen vermögen - darüber besteht keine Meinungsverschiedenheit - chemische Veränderungen an der Materie und auch an der lebenden Substanz zu erzeugen. Das Eiweiß, das den wesentlichsten Bestandteil der lebenden Substanz ausmacht, fängt erst in Blau an, das Licht zu absorbieren und wird besonders empfindlich im Ultraviolett. Das haben meine, das haben die oben angeführten Untersuchungen von Burge ergeben. Die ultravioletten Strahlen des Tageslichtes, diejenigen unserer intensiven, künstlichen Lichtquellen, erzeugen unter Umständen schwere Schädigungen an unserer Haut, an unseren Augen. Darüber besteht heute kein Zweife] mehr, wenn auch die Ansichten, wo diese Schädigungen beginnen, noch weit auseinandergehen. Der kleine Bruchteil der Sonnenenergie, der nach diesen Messungen der Physiker für die Wirkungen des Sonnenlichtes bei biologischen Vorgängen in Frage kommen kann, soll das Leben auf Erden schaffen und erhalten? Sollten da nicht Zweifel an der Richtigkeit jener Messungen berechtigt sein?

Als ich die erste Arbeit von Dorno, Studie über Licht und Luft in Hochgebirge, gelesen, kamen mir sofort Zweifel an der Richtigkeit seiner Beobachtungen. Als ich mir seine Versuchsanordnung genaner ansah, konnte ich zeigen, wo die Fehler liegen. Ich habe von ihm heftige Zurückweisung erfahren. In seiner letzten Entgegnung beklagt sich Prof. Dorno, dab ich ihm wiederholt nicht geantwortet habe. Der Grund liegt in dem Ton, in dem seine Ausführungen gehalten waren, und der mir eine weitere Diskussion unmöglich machte. Hier liegt zwischen den Feststellungen des Physikers und des Biologen ein großer Widerspruch, der zur Klärung drängt. Was ich an den Untersuchungen Dornos auszustellen habe, ist aus meinen früheren Arbeiten gu ersehen ${ }^{1}$ ). Heute möchte ich versuchen, den Widerspruch

1) Strahlentherapie 5, Hft. 1; Münch. med. Wochensohr. 1915, Nr. 48; Dtsch. med. Wochenschr. 1916, Ar. 20; Strahlentherapie 8, Graefes Areh. f. Ophthalmol.103. 
zu klären mit den Untersuchern, die bolometrische Messungen ihren Untersuchungen zugrunde gelegt haben. Die bolometrische Messung hat zur Voraussetzung, daß dabei die auftreffenden Strahlen in dem Körper, der sie restlos absorbiert, in Wärme umgewandelt werden. Als alle Strahlen restlos absorbierende Substanz verwendet man den Ruß. Soweit das sichtbare Spektrum reicht, mag dies angängig sein, wie es im Ultraviolett damit steht, wissen wir nicht. Ruß ist chemisch rein nicht darzustellen. Er ist ein ausgezeichnetes Adsorbens - die ultravioletten Strahlen können auch im Ruß und seinen Verunreinigungen chemische Veränderungen erzeugen. Diese chemischen Veränderungen sind auch mit Wärmeentwicklung verknüpft, aber diese Wärme läßt sich nicht in Vergleich stellen mit der Wärme am roten Ende des Spektrums.

Um festzustellen, wie weit bei den bolometrischen Messungen dem Ruß die Eigenschaft zukommt, alle Strahlen restlos in Wärme umzowandeln, habe ich bei meinen Arbeiten über die physikalischen Vorgänge bei der optischen Sensibilisation auch den Ruß auf seine lichtelektrische Zerstreuung geprüft (Pflügers Arch. f. d. ges. Physiol. 190, 311). Er zeigte außerordentlich lebhaft diese Reaktion und ließ dadurch erkennen, daß sehr intensive chemische Vorgänge in demselben durch Licht ausgelöst werden. Die Lichtstrahlen, die lediglich als Wärme wirken, vermögen keine lichtelektrische Zerstreuung zu erzeugen. Die Voraussetzung bei den bolometrischen Messungen, daß das Licht von Ruß restlos absorbiert und in gleiche Wärmemengen umgewandelt wird, ist im ultravioletten. Spektralteil nicht mehr zutreffend. Die oben ausgesprochenen Zweifel an den bisherigen Messungen der Sonnenenergie dürften daher berechtigt sein. 